\title{
Is there a correlation between the outcome of transurethral resection of prostate and preoperative degree of bladder outlet obstruction?
}

\begin{abstract}
Mi Mi Oh${ }^{1}$, Jin Wook Kim ${ }^{1}$ Je Jong Kim² and Du Geon Moon ${ }^{2}$
To compare the impact of transurethral resection of prostate (TURP) on symptom scores and maximal flow rates (Qmax) in patients with equivocal bladder outlet obstruction (BOO) and definite $\mathrm{BOO}$ and to assess the relationship between the surgical outcomes and degree of preoperative BOO, we prospectively evaluated men with lower urinary tract symptoms and bladder outlet obstruction index (BOOI) greater than 20, who were refractory to conventional medical treatment and underwent TURP. Urodynamic evaluation, International Prostate Symptom Score (IPSS), uroflowmetry, post-void residual volume (PVR) check and transrectal ultrasound were performed. $20<\mathrm{BOOI}<40$ was defined as equivocal BOO and BOOI $\geqslant 40$ as definite BOO. Changes of IPSS, Qmax, PVR and correlation analysis was performed between the degree of improvement of Qmax, subdomains of IPSS and BOOI. Fifty-four patients showed equivocal BOO and 80 patients showed definite BOO. Preoperatively equivocal BOO group and definite BOO group showed significant differences in maximal bladder capacity and prevalence of detrusor overactivity, whereas no difference was noted in prostate volume. Postoperatively both groups showed improvements in Qmax, obstructive (IPSSO) and irritative (IPSSI) subdomain of IPSS, but the degree of improvement in Qmax and IPSSI subdomain was statistically significantly greater in definite BOO group. The degree of improvement of Qmax and IPSSI showed weak correlation with preoperative BOOI. As a weak correlation was identified between preoperative degree of BOO and outcome of TURP, other factors other than BOOI such as severity of patients' symptoms should be considered in deciding treatment modality.
\end{abstract}

Asian Journal of Andrology (2012) 14, 556-559; doi:10.1038/aja.2011.157; published online 12 December 2011

Keywords: bladder outlet obstruction; bladder outlet obstruction index; International Prostate Symptom Scores

\section{INTRODUCTION}

Men with benign prostatic hyperplasia (BPH) can have benign prostatic enlargement (BPE), bladder outlet obstruction (BOO), lower urinary tract symptoms or a combination of these components. ${ }^{1}$ The relationships among urodynamic parameters, treatment outcome, symptom relief and patient satisfaction are still the subject of controversy. ${ }^{2}$ American Urological Association guidelines recommend to perform pressure flow studies in those who are planning to undergo surgical treatment without clear evidence of outlet obstruction $^{3}$ as the outcome of transurethral resection of prostate (TURP) seems to be related to the pre-treatment urodynamic finding. It has been reported that men with higher preoperative urethral resistance benefit more from $\mathrm{BOO}$ relief and invasive therapy in these patients may be optimal. But a problem rises when men with equivocal BOO who do not respond to or are not satisfied with the medical therapy. Hereto, only a few studies have been documented to assess the role of TURP on objective and subjective symptom improvement in men with equivocal BOO. Therefore, this study was performed to compare the impact of transurethral resection of prostate on changes of International Prostate Symptom Score
(IPSS) and maximal flow rate (Qmax) in men with equivocal $\mathrm{BOO}$ and definite $\mathrm{BOO}$ and to assess whether correlation exist between degree of $\mathrm{BOO}$ and degree of improvements in Qmax and IPSS subdomain scores.

\section{MATERIALS AND METHODS}

From April 2007 to April 2009, men with clinical BPH and bladder outlet obstruction index (BOOI) greater than 20 who were refractory to medical treatment and planned to undergo TURP were included in this study. Every patient who was planned for TURP underwent urodynamic studies preoperatively. Clinical BPH was defined by lower urinary tract symptom, BPE and/or suspicion of BOO in men aged 40 years or older. ${ }^{4}$ Patients with previous history of lower urinary tract or pelvic surgery, radiotherapy of pelvis and neurological disease were excluded from this study. Preoperatively digital rectal examination, transrectal ultrasound, uroflowmetry for Qmax, post-void residual urine volume (PVR), multichannel urodynamic study, with a pressure-flow study (MMS UD-2000; Medical Measurement System, Ennschede, The Netherlands), and IPSS were assessed. Detrusor overactivity was defined as involuntary detrusor contractions during

${ }^{1}$ Department of Urology, Korea University Medical Center, Seoul 435-766, Korea and ${ }^{2}$ Department of Urology, Korea University Medical Center and Korea University Institute of Regenerative Medicine, Seoul 435-766, Korea 
Table 1 Preoperative and postoperative variables according to bladder outlet obstruction index (BOOI)

\begin{tabular}{|c|c|c|c|c|c|c|}
\hline & \multicolumn{3}{|c|}{ Equivocal BOO group } & \multicolumn{3}{|c|}{ Definite BOO group } \\
\hline & Preoperation & Postoperation & $P$ value & Preoperation & Postoperation & $P$ value \\
\hline $\mathrm{Qmax}\left(\mathrm{ml} \mathrm{s}^{-1}\right)$ & $11.09 \pm 4.49 *$ & $16.38 \pm 5.36$ & 0.001 & $7.97 \pm 3.37$ & $16.44 \pm 5.75$ & 0.001 \\
\hline PVR (ml) & $55.80 \pm 72.46$ & $36.99 \pm 5.03$ & 0.102 & $74.23 \pm 82.95$ & $31.71 \pm 3.54$ & 0.941 \\
\hline IPSSI & $8.57 \pm 4.43$ & $3.49 \pm 0.47$ & 0.001 & $9.51 \pm 3.74$ & $4.51 \pm 3.22$ & 0.001 \\
\hline IPSSO & $12.75 \pm 5.65$ & $4.95 \pm 0.67$ & 0.001 & $14.36 \pm 4.50$ & $5.97 \pm 4.85$ & 0.001 \\
\hline Age (years) & $69.85 \pm 7.43$ & & & $69.94 \pm 7.53$ & & \\
\hline $\mathrm{DOA}(+)$ & $11^{*}$ & & & 30 & & \\
\hline PdetQmax & $41.15 \pm 15.40 *$ & & & $64.90 \pm 28.55$ & & \\
\hline Pdetmax & $51.01 \pm 12.10 *$ & & & $83.72 \pm 27.73$ & & \\
\hline MBC on $\mathrm{CMG}(\mathrm{ml})$ & $354.25 \pm 63.28 *$ & & & $316.57 \pm 88.34$ & & \\
\hline First sense (ml) & $191.69 \pm 87.40$ & & & $171.14 \pm 72.96$ & & \\
\hline Total volume (g) & $48.60 \pm 22.63$ & & & $51.70 \pm 22.78$ & & \\
\hline TZV (g) & $24.83 \pm 17.22$ & & & $29.11 \pm 18.13$ & & \\
\hline
\end{tabular}

Abbreviations: BOO, bladder outlet obstruction; CMG, cystometrogram; DOA, detrusor overactivity; PVR, post-void residual urine volume; IPSS, International Prostate Symptom Score; IPSSI, IPSS irritative subdomain score; IPSSO, IPSS obstructive subdomain score; MBC, maximal bladder capacity; PdetMax, maximal detrusor pressure; PdetQmax, maximal detrusor pressure at maximal flow rate; Qmax, maximal flow rate; TZZ, transitional zone volume.

$* P<0.05$, compared with preoperative data of definite BOO group.

cytometry, which might be spontaneous or provoked regardless of the amplitude according to the International Continence Society Classification (2002)..$^{5}$ Total IPSS was further divided into irritative (or storage) (questions 2, 4 and 7) and obstructive (or voiding) (questions 1, 3, 5 and 6) IPSS subscores. Urodynamic investigations and evaluations were performed according to the standards recommended by the International Continence Society. ${ }^{6}$ From the pressure-flow study, BOOI was defined as PdetQmax-2Qmax. ${ }^{7}$ Patients were divided into groups according to BOOI. We defined $\mathrm{BOOI} \geqslant 40$ as s definite $\mathrm{BOO}, 20<\mathrm{BOOI}<40$ as equivocal and $\mathrm{BOOI} \leqslant 20$ as normal. Postoperatively, 1 year after the surgery, IPSS, Qmax and PVR were assessed. Preoperative and postoperative parameters such as IPSS subdomain scores, Qmax and PVR were compared in each group as well as the degree of improvement in these variables were compared between the groups. Also analysis was performed to assess the correlation between the BOOI and degree of improvements in Qmax, PVR and IPSS subdomain scores. Variables were analyzed with Student's $t$-test, paired $t$-test and simple correlation analysis using the Statistical Package for the Social Sciences, version 13.0 for Windows (SPSS Inc., Chicago, IL, USA). $P<0.05$ was considered statistically significant.

\section{RESULTS}

Fifty-four patients showed equivocal BOO, whereas 80 patients had definite $\mathrm{BOO}$ on preoperative pressure flow study. General characteristics were shown in Table 1.

Patients with definite BOO showed smaller maximal bladder capacity $(316.57 \pm 88.34$ vs. $354.25 \pm 63.28, P=0.012)$ and higher prevalence of idiopathic detrusor overactivity (IDO) (30 vs. 11, $P=0.041$ ) compared with equivocal BOO. On linear by linear, positive correlation was noted between the presence of IDO and BOOI with $P=0.041$. IPSS irritative (IPSSI) $(9.51 \pm 3.74$ vs. $8.57 \pm 4.43, P=0.189)$ and IPPS obstructive (IPSSO) $(14.36 \pm 4.50$ vs. $12.75 \pm 5.65, P=0.071)$ subdomain scores and prostate volume $(51.70 \pm 22.78$ vs. $48.60 \pm 22.63$, $P=0.464$ ) did not show statistical differences between these groups. Postoperatively, both definite $\mathrm{BOO}$ group and equivocal $\mathrm{BOO}$ group showed improvements in Qmax, IPSSI and IPSSO subdomain scores (Table 1). The degree of improvements in Qmax (5.20 \pm 5.04 vs. $\left.8.47 \pm 6.52 \mathrm{ml} \mathrm{s}^{-1}, P=0.001\right)$ and IPSSI subdomain scores $(-3.59 \pm 3.61$ vs. $-5.01 \pm 3.79, P=0.032)$ were significantly greater in group with definite BOO. The degree of improvement in IPSSO did not show statistical difference $(-7.05 \pm 4.66 v s .-8.21 \pm$ 5.31, $P=0.197$ ) (Figure 1).

There was a weak positive correlation between preoperative degree of BOO and the degree of improvement in Qmax (Spearman rank test; $R=0.261, P=0.002$ ) (Figure 2a). Also, degree of BOO and the degree of improvements in IPSSI subdomain scores showed significant but weak negative correlation (Spearman rank test; $R=-0.186, P=0.032$ ) (Figure 2b).

\section{DISCUSSION}

Since the introduction of $\alpha$-blockers, physicians are rather reserved about resection of the prostate in equivocal or unobstructed men. ${ }^{2}$ It is well known that patients with definite BOO feel better after TURP and may benefit more compared with those without obstruction. But

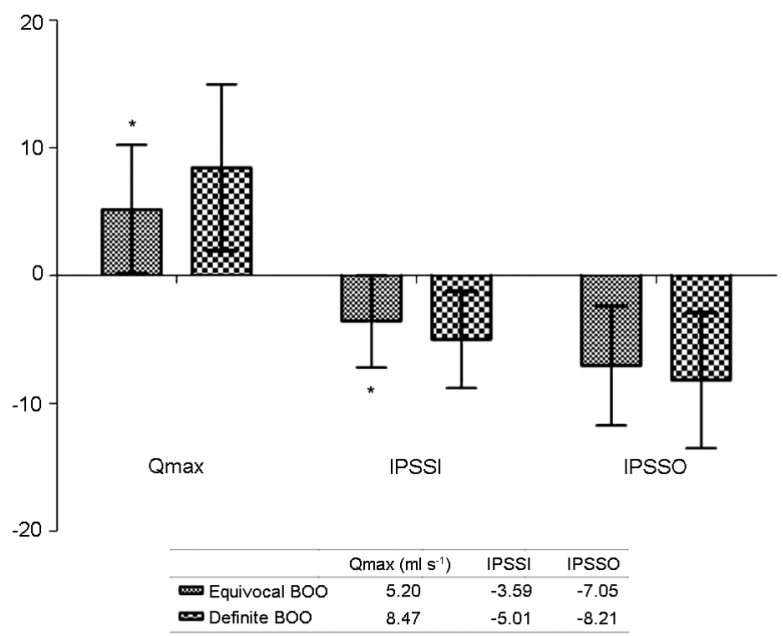

Figure 1 The degree of improvement in each parameter. ${ }^{*} P<0.05$, compared with definite BOO group. BOO, bladder outlet obstruction; IPSS, International Prostate Symptom Score; IPSSI, IPSS irritative subdomain score; IPSSO, IPSS obstructive subdomain score; Qmax, maximal flow rate. 

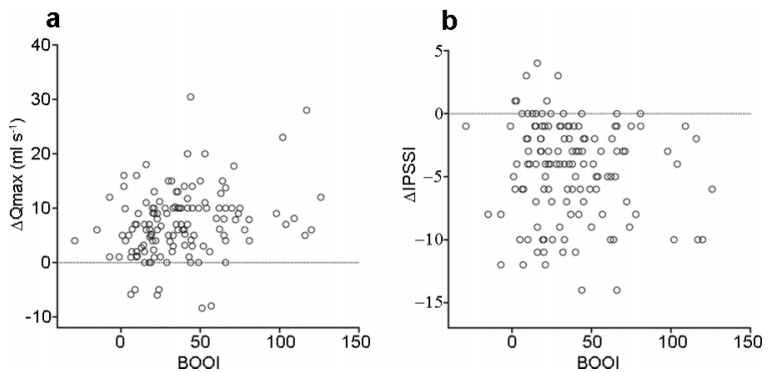

Figure 2 Relationship between BOOI and changes Qmax and IPSSI scores. (a) With increasing BOOI, the degree of improvement of Qmax increased as well but showed a weak correlation ( $P=0.002$, Pearson's $R=0.261$ ). (b) Degree of BOOI and the degree of improvement in IPSSI domain showed significant but weak negative correlation ( $P=0.032$, Pearson's $R=-0.186$ ). BOOI, bladder outlet obstruction index; IPSS, International Prostate Symptom Score; IPSSI, IPSS irritative subdomain score; Qmax, maximal flow rate.

physicians often face patients with equivocal BOOI who do not benefit from or tolerate medication and warrant surgery.

In our prospective study, we identified that those with definite $\mathrm{BOO}$ showed smaller maximal bladder capacity with higher prevalence of IDO $(P=0.041)$. Also using linear-by-linear association test, we identified a positive correlation between degree of $\mathrm{BOO}$ and presence IDO in patients with BOOI greater than 20. No differences were shown in prostate volume (Table 1). This finding was in accordance with our previous study which revealed a positive correlation between the presence of IDO and BOOI. ${ }^{8}$ There was an increasing probability of IDO with increasing BOOI as shown in linear-by-linear association test $(P=0.041)$, confirmed by a recent report by Oelke et al. ${ }^{9}$ They demonstrated that with increasing BOO grade, there was an increasing probability of detusor overactivity and tendency toward an earlier appearance and higher amplitude of detusor overactivity.

It was noteworthy that postoperatively both equivocal BOO group and definite BOO group showed significant improvements in Qmax, IPSSI and IPSSO subdomain scores. This finding was consistent with the report by van Venrooij et al. ${ }^{10}$ demonstrating that men who did not have obstruction preoperatively or were in equivocal condition also benefited from resection. Improved symptom problems, decreased impact of bother and improved quality of life were significantly associated with improved bladder outlet function. Although no difference was noted in prostate volume between those with equivocal BOO and definite $\mathrm{BOO}$, the mean prostate volume was $48.60 \pm 22.63$ in group with equivocal BOO. This finding showed that not only BOOI and prostatic enlargement were important in treatment decision. The degree of improvement in Qmax and IPSSI subscores were superior in group with definite BOO. But this finding may be originated from the fact that the baseline values in Qmax was lower and IPSS subscores were higher in definite BOO group compared with equivocal BOO. It was reported that patients with BOO showed higher success rate for TURP than those without it in various studies. ${ }^{11,12}$ van Venrooij et al. ${ }^{10}$ reported that the reductions in symptom and bother in the equivocal and unobstructed men were approximately $70 \%$ of those in the obstructed men. They also documented in other study that improvement in obstruction grade, symptom index, symptom problem index, BPH impact index and quality of life were related to preoperative obstruction grade. Recently Tanaka et al. ${ }^{13}$ reported comparable outcome in IPSS, quality of life index and PVR 3 months after TURP among different degree of BPE. ${ }^{13}$ Even in patients without obvious
BOO, a promising effect of TURP was observed in their study. The reason for this outcome was unclear, but removal of $\mathrm{BOO}$, irrespective of the degree of $\mathrm{BOO}$, may relieve symptomatic and urodynamic improvements. Relief of BOO has been shown to have beneficial effect on IPSSI subscores by means of decrement of instability and increment of effective capacity. It is proposed that symptoms of nocturia, frequency, and urgency and associated symptoms also strongly decrease after relief of BOO and it is likely that irritative effects not due to BOO cause symptoms in BPE cases. Also, effective bladder capacity partly caused by improved bladder emptying after transurethral prostate resection contributes to a significant decrease in symptoms and bother, and to improved well-being. ${ }^{10}$ This study had limitation in the point that study population is limited to those who had undergone surgical treatment and who were refractory to medical treatment and relatively had more severe symptoms, so this study may have the possibility of selection bias.

We must emphasize that significant but weak correlation was noted between the degree of improvements in Qmax (Pearson's $R=0.261$, $P=0.002$ ) and IPSSI subscores (Pearson's $R=-0.186, P=0.032$ ) with preoperative degree of $\mathrm{BOO}$ (Figure 1). This finding implied that although preoperative BOOI can be a predictor of surgical outcome, as the correlation between the degree of improvement of Qmax and IPSSI score was weak, we should consider TURP as the treatment modality in those who do not warrant medical treatment at least in patients with equivocal BOO.

\section{CONCLUSIONS}

Definite BOO showed greater improvement compared with patients with equivocal BOO. As a weak correlation was identified between preoperative degree of $\mathrm{BOO}$ and outcome of TURP, other factors other than BOOI such as severity of patients' symptoms should be considered in deciding treatment modality.

\section{AUTHOR CONTRIBUTIONS}

MMO participated in the design of the study and drafted the manuscript. JWK performed the statistical analysis. JJK and DGM conceived of the study, and participated in its design and coordination and helped to draft the manuscript.

\section{COMPETING FINANCIAL INTERESTS}

The authors declared no conflict of interest.

\section{ACKNOWLEDGMENTS}

This work was supported by Korea University Grant (K1131741).

1 Madersbacher S, Alivizatos G, Nordling J, Sanz CR, Emberton M et al. EAU 2004 guidelines on assessment, therapy and follow-up of men with lower urinary tract symptoms suggestive of benign prostatic obstruction (BPH guidelines). Eur Urol 2004; 46: 547-54

2 van Venrooij GE, van Melick HH, Boon TA. Comparison of outcomes of transurethral prostate resection in urodynamicallyobstructed versus selected urodynamicallyunobstructed or equivocal men. Urology 2003; 62: 672-6.

3 McVary KT, Roehrborn CG, Avins AL, Barry MJ, Bruskewitz RC et al. Update on AUA guideline on the management of benign prostatic hyperplasia. J Urol 2011; 185: 1793-803.

4 Wadie BS, Ebrahim el-HE, Gomha MA. The relationship of detrusor instability and symptoms with objective parameters used for diagnosing bladder outlet obstruction: a prospective study. J Urol 2002; 168: 132-4.

5 Abrams P, Cardozo L, Fall M, Griffiths D, Rosier P et al. The standardisation of terminology of lower urinary tract function: report from the Standardisation Subcommittee of the International Continence Society. Am J Obstet Gynecol 2002; 187: 116-26. 
6 Ameda K, Koyanagi T, Nantani M, Taniguchi K, Matsuno T. The relevance of preoperative cystometrography in patients with benign prostatic hyperplasia: correlating the findings with clinical features and outcome after prostatectomy. J Urol 1994; 152: 443-7.

7 Rosier PF, de la Rosette JJ, Wijkstra H, van Kerrebroeck PE, Debruyne FM. Is detrusor instability in elderly males related to the grade of obstruction? Neurourol Urodyn 1995; 14: 625-33.

8 Oh MM, Choi H, Park MG, Kang SH, Cheon J et al. Is there a correlation between the presence of idiopathic detrusor overactivity and the degree of bladder outlet obstruction? Urology 2011; 77: 167-70.

9 Oelke M, Baard J, Wijkstra H, de la Rosette JJ, Jonas $U$ et al. Age and bladder outlet obstruction are independently associated with detrusor overactivity in patients with benign prostatic hyperplasia. Eur Urol 2008; 54: 419 26
10 van Venrooij GE, van Melick HH, Eckhardt MD, Boon TA. Correlations of urodynamic changes with changes in symptoms and well-being after transurethral resection of the prostate. J Urol 2002; 168: 605-9.

11 Rollema HJ, van Mastrigt R. Improved indication and followup in transurethral resection of the prostate using the computer program CLIM: a prospective study. J Urol 1992; 148: 111-5discussion 5-6.

12 Robertson AS, Griffiths C, Neal DE. Conventional urodynamics and ambulatory monitoring in the definition and management of bladder outflow obstruction. J Urol 1996; 155: 506-11.

13 Tanaka Y, Masumori N, Itoh N, Furuya S, Ogura H et al. Is the short-term outcome of transurethral resection of the prostate affected by preoperative degree of bladder outlet obstruction, status of detrusor contractility or detrusor overactivity? Int J Urol 2006; 13: 1398-404. 